Shaviv, 1994). We note that the new opacity data bases are presently only of limited value for the modeling of accretion disks since the tables do not provide data for the low temperatures and densities required. The addition of tables from other sources in the missing range is hardly possible since essentially all solutions for the structure equations require the continuity of high order derivatives. However, it is to be expected that in the near future both the application of solutions for boundary value problems which take switching points explicitly into account (Bock,1994) and the development of adaptive schemes for the solution of the multidimensional radiative transfer equation on parallel computers (Fuhrer, 1994; Kanschat, 1994) will lead to a significant improvement of the situation.

Adam, J.; A\&A 240, 541 (1990)

Adam, J., Storzer, H., Shaviv, G., Wehrse, R.; A\&A 193. L1 (1988)

Baschek, B., Papkalla, R., Wehrse, R.; Proc. 2nd Technion Haifa Conf., Regev, O., Shaviv, G., eds., p. 176 (1993)

Bock, H.G.; Proc. R1.06 Carl-Cranz-Gesellschaft (1994)

Frank, J., King, A., Raine, D.; Accretion Power in Astrophysics, Cambridge University Press (1992)

Fuhrer, C., Proc. Workshop on Numerical Methods for Multidimensional Radiative Transfer, Heidelberg, in press (1994)

Kanschat, G, Proc. Workshop on Numerical Methods for Multidimensional Radiative

Transfer, Heidelberg, in press (1994)

Shaviv, G., Wehrse, R.; A\&A 251, 117 (1991)

Wehrse, R., Baschek, B., Shaviv., Interacting Binary Stars, Shafter, A.W., ed., ASP Conf. Ser. 56, p.35 (1994)

Wehrse, R., Proc. Workshop on Numerical Methods for Multidimensional Radiative

Transfer, Heidelberg, in press (1994)

Wheeler, J.C., Ed., Accretion Disks in Compact Stellar Systems, World Scientific (1993)

\title{
OPACITIES OLD AND NEW IN WHITE DWARF MODELS
}

\section{STEVEN D. KAWALER}

\section{Department of Physics and Astronomy, Iowa State University,} Ames, LA 50011 USA

The new radiative opacity compilations by the Opacity Project (OP) and the Livermore group (OPAL) represent a significant advance in the accuracy of this constitutive quantity. White dwarfs present a useful medium for the analysis of the opacities in regions of the density-temperature-composition phase space that are not sampled by any other stars. The bulk of the interior of white dwarfs is degenerate, and energy transport is therefore through conduction by electrons. This efficient energy transport mechanism results in a nearly isothermal core with temperatures in excess of 10 million $\mathrm{K}$. The cooling of this core 
is controlled by the thermal properties of the nondegenerate outer layers, where heat is transferred either by radiation or, in cool white dwarfs, convection.

The contact points between the opacities and observations are the white dwarf luminosity function and white dwarf pulsations. We discuss preliminary investigations of how the new opacities affect research on these two problems. In doing so, we also show where further tabulations of opacities and equations of state are vital.

The white dwarf luminosity function is used in studies of the age and star formation history of our galaxy. Models of cooling white dwarfs that are instrumental in this work rely on radiative opacities. The cooling of white dwarf stars is sensitive to the opacity of relatively pure helium, and to a lesser extent hydrogen. In white dwarfs with effective temperatures between $25,000 \mathrm{~K}$ and $10,000 \mathrm{~K}$, much of the heat transport in the outermost layers is by convection, but radiative transport is important, too. In evaluating the effect of new opacities, we express the effect in terms of the change in the age (in Gyr) of the galactic disk as determined using the coolest white dwarfs and cooling models. The new opacities are, on average, slightly larger than the old opacities at the temperatures and densities of interest. We use a simple model of white dwarf cooling (one which assumes radiative transport in the outer layers) to find an upper limit for the effect that slightly larger opacities have on the cooling age of white dwarfs. For an increase in opacity of $25 \%$, the simple cooling models indicate that the cooling age would increase by up to $0.5 \mathrm{Gyr}$. However, using much more realistic cooling calculations by Wood (1991), we show that the increase in the cooling age is only 0.1 to $0.2 \mathrm{Gyr}$. Similar calculations by D'Antona and Mazzitelli suggest that the effect of such an opacity increase would only increase the age by $0.05 \mathrm{Gyr}$. Our conclusion is that the new opacities have negligible effect on theoretical models of white dwarf cooling and the results from their comparison with the observed white dwarf luminosity function.

The main need in the use of the new opacities in modeling cooling white dwarfs is phase-space coverage. The opacity of stellar matter at low temperatures and high densities, as pointed out by D'Antona and Mazzitelli (1990), is very important for white dwarf models. Unfortunately, the OPAL opacity general distribution does not cover the conditions found over a significant portion of white dwarf envelopes. Such data would be extremely welcome.

The new opacities result in little or no differences in the pulsation properties of DO or DB white dwarfs. Both the adiabatic (pulsation periods) and nonadiabatic (growth rates and damping times) properties of the models are slightly altered. However, with the new capabilities of the atomic physics collaborations, a significant improvement in the accuracy of these calculations is possible. The pulsation properties are influenced by a sensitive balance between equation of state properties and opacity. At this time, most computations of variable star pulsation and structure use a different formulation for the equation of state than the producers of opacity tables. Key quantities are the opacity derivatives and the adiabatic gradients. Stellar structure calculations use opacities from the tables, numerical opacity derivatives using the tables, and equation of state derivatives from their own 
individual formulations. Thus there is a fundamental inconsistency (however small) between the opacities and equation of state quantities that are essential for pulsation analysis.

This problem could be removed if the opacity groups could provide the equation of state data that they used in construction of the opacity tables. Similarly, separate tables with opacity derivatives would give far more stable and self-consistent pulsation results if those derivatives are provided as primary quantities and not as derived by numerical differentiation from the supplied tables.

\section{LIST OF POSTER PAPERS:}

1. Atomic Data for Bound-Bound and Bound-Free Opacities of Hydrogen in a Strong Magnetic Field

N. Merani, J. Main, G. Wunner (Lehrstuhl fur Theoretische Physik I, Ruhr-Universitat Bochum)

2. Influence of Nonideal Effects of Dense Plasma on Solar Interior Opacity

G. Luo (Yunnan Observatory, Chinese Academy of Sciences, Kunming, P.R. China)

3. Low Temperature Rosseland Opacities

D.R. Alexander (Physics Department, Wichita State University, Wichita, KS 67260, U.S.A.)

4. Stellar Opacities for Very Low Mass Stars and Brown Dwarfs

Takashi Tsuji and Keiichi Ohnaka (Institute of Astronomy, The University of Tokyo, Mitaka, Tokyo, 181 Japan)

5. Low-Temperature Opacities Applied to Solar Calibrated Models

C. Neuforge (Institut d'Astrophysique de l'Universite de Liege, 5 Avenue de Cointe, B-4000 Liege,

Belguim), J. Provost, G. Berthomieu, P. Morel (Department Cassini, Observatoire de la Cote d'Azur, BP 229, 06304 Nice Cedex 4, France)

6. Atomic Structure Calculations and New Los Alamos Astrophysical Opacities N.H. Magee, J. Abdallah, Jr., R.E.H. Clark, J.S. Cohen, L.A. Collins, G. Csanak, C.J. Fontes, A. Gauger, J.J. Keady, D.P. Kilcrease and A.L. Merts (Theoretical Division, Los Alamos National Laboratory)

7. Critically Evaluated Data and Databases on Atomic Spectra-Energy Levels, Wavelengths, Transition Probabilities, Shape Parameters

J. Fuhr, D. Kelleher, W.C. Martin, A. Musgrove, J. Sugar and W.L. Wiese (National Institute of Standards and Technology (NIST), Gaithersburg, MD 20899, USA)

8. Analytic Fits for the Partial Photoionization Cross-Sections

D.G. Yakovlev (Ioffe Institute of Physics and Technology, St. Petersburg, Russia) and D.A. Verner (Kapteyn Astronomical Institute, Groningen, The Netherlands)

9. Atomic Data for Absorption Lines from the Ground Level at Wavelengths Longward of $228 \AA$ D.A. Verner and P.D. Barthel (Kapteyn Astronomical Institute, Groningen, The Netherlands) and 